\title{
FLECHA EN FORJADOS
}

\author{
(FLOOR SLAB SAG)
}

\author{
José Luis de Miguel, Arquitecto. \\ Catedrático de Estructuras de ARQUITECTURA en Valladolid
}

\section{RESUMEN}

Para el cálculo de la flecha en piezas de hormigón armado, basta integrar la curvatura de sus diversas secciones. La curvatura se expresa como cociente de deformación de uno cualquiera de los bordes - siendo más fácil hacerlo con el traccionado - entre la fracción de canto que mide la distancia del borde a la fibra neutra. La estimación de deformación en tracción puede hacerse a partir del momento, brazo de palanca, y sección de armadura.

La flecha como - doble- integración de la curvatura se obtiene, en piezas simétricas sustentadas en ambos extremos, como producto de dilatación media por la fracción de luz que representa la distancia entre centro de gravedad del diagrama de deformaciones de media luz, al extremo de la pieza.

En definitiva, se puede establecer que, para una configuración dada, la flecha es proporcional a la dilatación máxima del acero y la relación de la luz al canto de la pieza. El factor numérico de la expresión recoge la relación de dilatación media a máxima (entre 0,50 y 1,00), la de fracción de luz entre el centro de deformaciones y el extremo (que varía correlativamente con lo anterior entre 0,33 y 0,25), y la fracción de canto traccionado, del orden del $50 \%$ en piezas de sección rectangular y hasta $90 \%$ en sección en $T$. La existencia de armadura sobreabundante reduce la flecha casi en forma proporcional. El factor numérico debe tener en cuenta la incidencia de las partes no fisuradas, que cuando la flecha es critica, es relativamente baja.

\section{SUMMARY}

To calculate the sag in pieces of reinforced concrete, it is only necessary to integrate the curve of its various sections. The curve is expressed as the deformation quotient of any one of the edges -it is easier to do it with one under tension - between that fraction of the edge which is the distance from the edge to the neutral fibre. Deformation under tension can be estimated on the basis of the moment, leverage and section of reinforcement.

Deflection as a double integration of the curve is obtained, in symmetrical pieces supported at both ends, as the product of average expansion multiplied by the fraction of span represented by the distance between the centre of gravity of the average-span strain diagram and the end of the piece.

It can be said definitively that, for a given configuration, sag is proportional to the maximum expansion of the steel and the relation of the span to the edge of the piece. The numerical factor of the expression includes the relation between average and maximum expansion (from 0.50 to 1.00), that of the fraction of span between the centre of deformation to the end (which varies correlatively to the previous figures between 0.33 and 0.25 ), and the fraction of edge under tension, around $50 \%$ in rectangular-sectioned pieces and up to $90 \%$ in $T$ sections. If there is surplus reinforcing this reduces sag almost proportionately. The numerical factor must take account of the incidence of non-fissured parts, which, when deflection in critical, is relatively low.

\section{Introducción}

La flecha en forjados es una variable relevante. Al diseñar un forjado, la mejor elección conduce inevitablemente, en la práctica totalidad de los casos, a disponer el menor canto compatible con una flecha tolerable. Por debajo de ese valor, la armadura - al disminuir el brazo de palanca y existir la necesidad de bajar la tensión - aumenta tan rápidamente que el resultado no es competitivo. Por encima, el aumento de peso propio, y del material empleado en hormigón y bovedillas, compensa la disminución de armadura.
La importancia del tema ha supuesto un tratamiento insistente en la literatura técnica, de lo que es buena muestra el documento adjunto, en el que los personajes creados por Galileo, en su lenguaje arcaico, debaten cómo puede establecerse una fórmula comprensiva del problema.

Hemos intentado en la traducción, respetar la terminología original, en la esperanza de que el lector pueda llegar a entenderlo por sí mismo, sin necesidad de añadir acotaciones para definirlos, o de cambiarla por las palabras que se usan en la actualidad para desig- 
nar parecidos conceptos, y esperamos que comprenda el sentido que tiene.

Esta versión que se publica ahora, circuló en forma restringida en las Navidades de 1985, año en que tuvimos acceso al original.

\section{JORNADA XXXIII}

\section{De la deflexión en piezas destinadas a forjar pisos}

Salviati._La jornada de ayer la habiamos destinado a cómo las piezas deben poseer suficiente resistencia, pero no es menos importante que posean suficiente indeformabilidad para no acusar excesiva deflexión; y a ese cometido destinaremos la de hoy.

Sagredo.-¿YY dónde es más pertinente dicha cualidad?

Salv.-Sobre todo en las piezas destinadas a forjar pisos, de forma que no se dañen los revocos ni enlucidos, y menos aún las albañilerias, que con frecuencia son más sensibles, sobremanera las realizadas con morteros de cemento sin adoptar la práctica antes habitual de bastardearlos con cal. Las muchas deflexiones desarman los suelos y mueven los recibidos de puertas y demás carpinterías; $y$, lo que es peor, con el cansancio natural de los materiales - notable en la madera y el hormigón-, se acrecientan tales movimientos con el tiempo, de forma que los desdoros se manifiestan incorregibles y graves cuando ya no hay a quien presentar las justas quejas. Por lo que será virtud del que discierne las piezas del forjado tomar las precauciones contra ello.

Simplicio.- ¿Pero no es cierto que cuando la pieza es robusta y fuerte no hay deflexiones?

Salv.-No es tal, sino que cuando la pieza es robusta como decís, las deflexiones son tan pequeñas que no se perciben fácilmente, y su poca magnitud es la señal de bondad. Pero si miráis con detenimiento o enfiláis la pieza de costado la notaréis, y es más, crecerá cuando se carga la pieza, y menguará cuando se la descarga, y, aunque el paso del tiempo mantenga o acreciente su valor, la descarga volverá a reducirla.

Sagr.-Y ¿cómo obtendremos su valor?, porque colijo que tendrá que ver con todo lo anterior, como luz y carga de la pieza, pero también de su porte y del tipo de material con que la hagamos, que no será igual de hierro que de madera o de hormigón, aunque los esfuerzos sí lo son.
Salv.-En siendo lo buscado algo geométrico, y por adquirir soltura en ello, lo intentaremos con la geometría, rindiendo homenaje a nuestro maestro Euclides.

Sagr.-Agradeceremos ese procedimiento, ya que la geometria se ve y las demostraciones y formulaciones son menos convincentes.

Salv.-Comenzaremos entonces por casos de geometría sencilla, y proseguiremos tomando sólo una cuestión nueva cada vez, de forma que nuestro razonamiento no se vea impelido a salvar fuertes escollos.

Sea pues una pieza simple, de material elástico, en el que las dobles tensiones ocasionan dobles dilataciones, y lo mismo en dilatación que en contracción, y tomaremos una pieza cuya sección recta no cambie en todo su largo, como es lo habitual en vigas de techo, como las rectangulares, cuyo centro de gravedad está en la mitad del canto, y consideraremos la siguiente.

\section{Proposición I}

Una pieza, de sección constante y simétrica, y material elástico, sometida en toda su longitud a una única flexión, simplemente presentada en sus dos extremos, posee una deflexión - relativa a la semiluz-directamente proporcional a la dilatación máxima y a la esbeltez, o relación de su largura a su canto.

Sea la pieza del grabado que os muestro, y considérense los puntos aa' y bb' antes de cargar la pieza en cortes rectos, siendo $a a^{\prime}=b b^{\prime}$.

Sabemos que la flexión reduce o acorta la distancia de la parte en compresión, mientras que aumenta la tensionada, por lo que después de la carga, ab no será paralelo a a'b', y si es único el valor de flexión en todo punto, igual será el acortamiento y estiramiento, por lo que la figura deformada no podrá ser otra que una línea curvada igual en todos los puntos, es decir, una circunferencia.

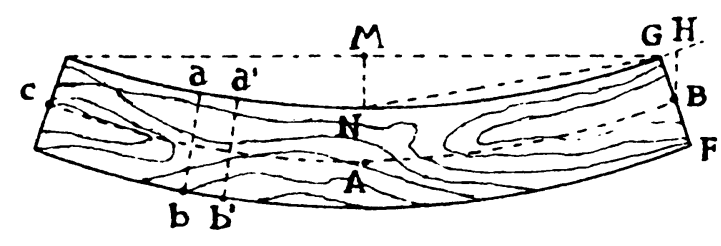

I

Trazando por el extremo B una línea vertical, BH, la medida GH no es sino la contracción de toda la parte superior NG, y el valor $M N$ no es otro que la deflexión buscada.

http://informesdelaconstruccion.revistas.csic.es 
Como el ángulo MGN es inscrito a la circunferencia, es mitad del arco abarcado $C A B$, que no es otro que el ángulo GBH, y como ambos son pequeños, o así intentamos que lo sean, y tomando para el valor NG la mitad de la luz, podemos concluir que la relación de la deflexión a semiluz es la mitad que el alargamiento a la mitad del canto.

También podemos expresarlo como que la relación de deflexión a dilatación de semiluz, es la mitad de la relación de medio canto a la semiluz.

Si la fibra superior $\mathrm{N}$ acorta una cantidad e por cada unidad de longitud, el alargamiento total será tantas veces superior a e como unidades posea la semiluz, lo que puede expresarse como:

$$
\frac{M N}{e \cdot A B}=\frac{1}{2} \frac{A B}{B G} ; \quad \frac{M N}{A B}=\frac{e}{2} \frac{A B}{B G}
$$

o bien:

$$
\frac{\delta}{L / 2}=\frac{e}{2} \frac{L}{h}
$$

por lo que podemos afirmar que la deflexión relativa a la semiluz es proporcional a la dilatación e y a la esbeltez L/h, y que dicha razón es 1/2. C.Q.D.

Vemos pues que si usamos un material en el que, para la resistencia máxima, se da un alargamiento de una milésima, una pieza elegida de una fortaleza justa poseerá en su cara inferior ese alargamiento; y con un canto décimo de la luz poseerá una deflexión de un medio por un milésimo por diez, o sea de un doscientosavo; y esa cantidad es la que la prudencia nos dice que es razonable para los suelos de pisos.

Sagr.- ¿Y por qué hablais de la deflexión relativa a la semiluz?

Salv.-Porque la magnitud relativa es la que interesa. Si en un dintel o cargadero una deflexión liviana rompe y acusa el marco con el tímpano superior, una viga que salve dos o tres veces más luz podrá descender doble o triple dimensión, sin por ello perjudicar en mayor medida a un tabique que sobre ella repose; ya que lo que daña el revoco no es lo que desciende -el dintel-que si descendiera todo por igual no lo sentiría; lo gravoso es que de dos puntos próximos, desciendan uno mucho más que otro; que lo que importa es cuánto descienden entre sí para lo separados que estén, y con la semiluz mediremos mejor dicha diferencia, que valdrá además para aleros y balcones.

Sagr.-De lo que decís, se deduce que si salva una luz diez veces superior podrá, sin desdoro de los demás elementos, descender una cantidad diez veces superior.
Salv.-En efecto, pero la cautela nos pide ser más prudentes cuanto mayor es la luz, y que los razonamientos son simples en situaciones simples y no se deben llevar las conclusiones a puntos muy alejados de los de partida. Lo que algunos códigos recomiendan a partir de una luz es rebajar a la mitad las deflexiones de cuanto exceda dicha luz; y aún en ocasiones, para otra luz mayor, detener por completo el valor de la deflexión tolerable.

Sagr.-A lo que se ve, si la fortaleza es justa, sólo la esbeltez explica la deflexión.

Salv.-Bien decís, y lo podemos expresar como:

- De dos jácenas del mismo material, jácenas elegidas con fortaleza justa para la carga, las flechas relativas son proporcionales a sus esbelteces.

O bien:

- De dos vigas de la misma esbeltez y material, las flechas relativas son proporcionales a las dilataciones máximas, o a la resistencia fijada para calcularlas.

Sagr.-De lo que se deduce que la mejor manera de mantener acotadas las deflexiones es hacerlo con la esbeltez, de forma que para más luz vengamos en destinar proporcionalmente más canto.

Salv.-Pero tal conclusión no puede llevarse a toda luz por grande que sea, porque si nos fijamos un valor de deflexión, ésta aumenta para un canto dado y un material justamente aprovechado en su fortaleza, con la segunda potencia de la luz, de forma que a doble luz hay cuadruple deflexión, y ésa y no otra es la limitación que existe para que no sea posible cubrir luces indefinidamente grandes.

Aunque simple es la Proposición I, no debe manejarse sin reflexión, y para ilustrarlo veamos qué sucedería en una pieza de escuadría rectangular de doble ancho que canto, al disponerla por tabla en vez de por canto.

Simp.-Al ser el canto mitad, la flecha relativa también sería mitad.

Sagr.-Pero olvidáis que la fortaleza habrá disminuido también.

Simp.-Habrá pues que suponer que antes tenia fortaleza sobrante, que lo que nos pregunta el señor Salviati es acerca de la deflexión.

Salv.-Pero, si tenía fortaleza sobrante, poseía menor tensión y, por tanto, menor deformación, que el Línceo http://informesdelaconstruccion.revistas.csic.es 
nos demuestra (1) que en una pieza como la que hablamos, la fortaleza por canto es doble que por ancho, ya que es directamente proporcional al ancho y al cuadrado del canto, por lo que, al cambiarla de posición, doblaría su tensión, y, aunque no rompiera, su alargamiento seria también doble, y como doble es su esbeltez, sería de cuadruple deflexión. De lo que se deduce que la proposición demostrada debe manejarse con cuidado, no sea que aplicándola a la ligera arroje resultados engañosos.

Pero no extraigamos más conclusiones, ya que resulta difícil concebir una carga que ofrezca flexión única, y tomemos una pieza cargada con un sólo peso en el centro, admitiendo que la propia jácena no posee peso apreciable, para ver en ese caso cómo es la deflexión.

Sagr.-Os ruego que me dejéis intentarlo. La flexión es ahora máxima en el centro, y nula en los extremos, por lo que la pieza será más curva en su parte central, y varía gradualmente la dilatación desde el centro hasta los extremos, estando el valor medio de la dilatación en dicho centro. Como por cada punto que supere la dilatación media, existen otros dos, uno que no llega a dicho valor, y otro que lo sobrepasa, a igual distancia y a ambos lados, podremos utilizar la Proposición I sin más que tomar la dilatación media, en vez de la máxima.

Salv.-No os fiéis de razonamientos tan simples, que a fuerza de claros parecen ser ciertos; más bien proceded con mucho tiento, que la cuestión es delicada.

Sean pues las dos piezas del grabado siguiente.

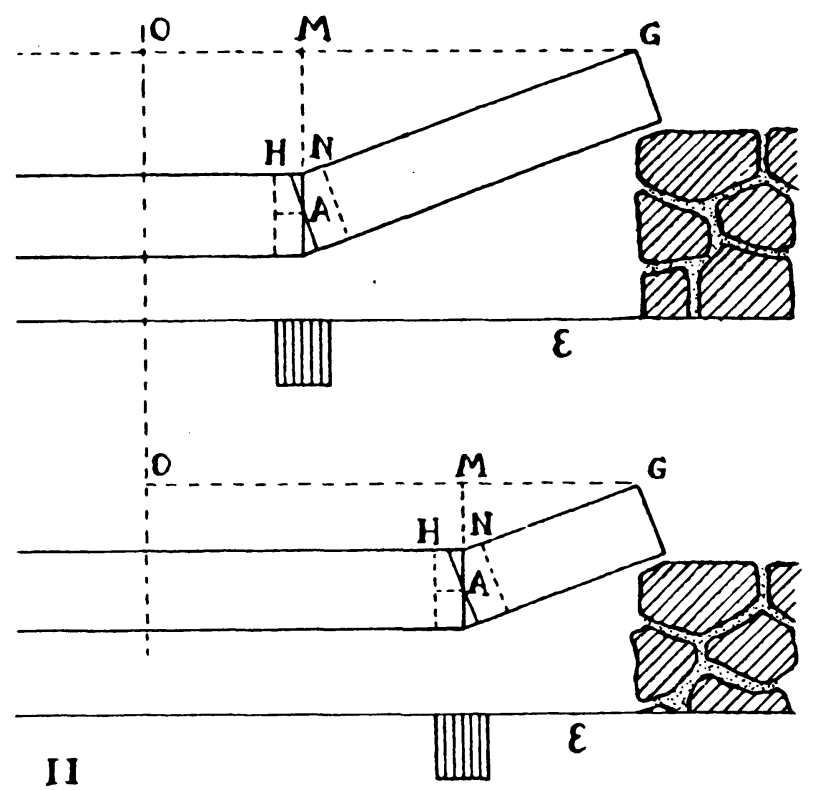

(1) Se refiere a "Dos nuevas Ciencias". JORNADA SEGUNDA. Proposición II (1938).
Supongamos que en cada una existe sólo una pequeña parte con flexiones, de forma que podremos admitir, sin más que suponer que es muy pequeña, que todo el alargamiento se produce en su centro, dejando todo el resto de la pieza sin deformación alguna.

De la semejanza de los triángulos MNG y HNÄ, se deduce que el alargamiento es la mitad del canto, como la deflexión es a la distancia de la zona en flexión al apoyo, y, por tanto, si dicha distancia es menor, también lo es y proporcionalmente la deflexión, y a la tal distancia que da tanta deflexión como cuanto mayor sea su valor, y por paralelismo con el vuelo de esfuerzos, la denominaremos vuelo de deflexión.

Sagr.-Entonces, no será posible establecer para este caso la conclusión general, puesto que cada tipo de carga exige una curva distinta, y sus propiedades serán asimismo diferentes, debiendo establecer, para cada una, conclusiones que sólo valdrán para un sólo caso de carga.

Salv.-Veamos si la geometría puede ayudarnos en ello, porque alguna propiedad será la general.

Si la pieza poseyera dos alargamientos en dos zonas distintas, mientras éstos sean pequeños $-\mathrm{y}$ los valores a los que pensamos circunscribirnos así lo indican-, pueden sumarse los ángulos en el apoyo y las deflexiones, de forma que la deflexión total depende de cada alargamiento en razón a su distancia al apoyo, es decir, del momento de primer orden del alargamiento.

Para obtener el total de la deformación de entrambas, basta proceder con el total del alargamiento, como si se produjera todo él en el centro de gravedad del alargamiento real. $Y$ lo mismo si son dos o varias, o si el alargamiento varía continuamente, que esa es la propiedad del centro de gravedad, dar el mismo momento de primer orden.

Podemos, pues, resolver cualquier variación de dilataciones, sin más que obtener de ellas el centro de gravedad de su distribución y suponer alli todo el alargamiento. La deflexión será pues, de acuerdo con el siguiente grabado:

$$
\frac{\delta}{\mathrm{v}}=\frac{\triangle}{\mathrm{h} / 2}
$$

y como el alargamiento total es igual al alargamiento medio por la distancia total -o más bien esa será en adelante la definición de! alargamiento medio-, quedaría:

$$
\frac{\delta}{\mathrm{v}}=\mathrm{e}_{\mathrm{m}} \frac{\mathrm{L} / 2}{\mathrm{~h} / 2}:: \frac{\delta}{\mathrm{v}}=\mathrm{e}_{\mathrm{m}} \frac{\mathrm{L}}{\mathrm{h}}
$$

con lo que queda demostrado dicho aserto. 


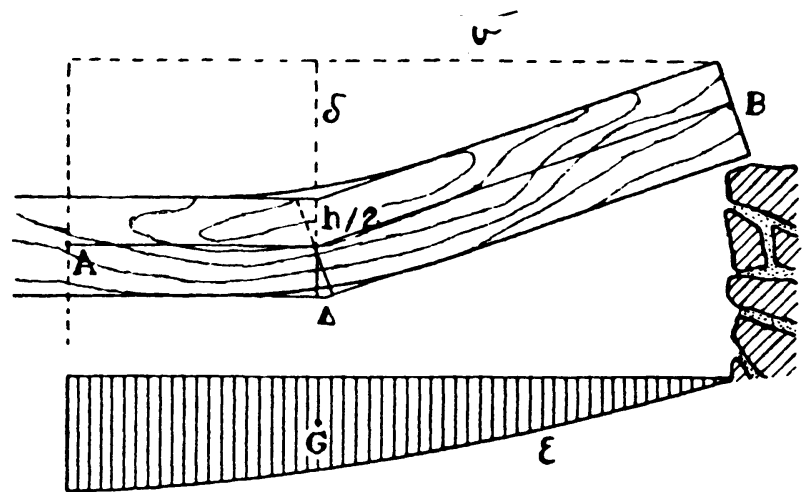

J11

Sagr.- ¿Y no es lo mismo? Pues seguimos obteniendo la deflexión relativa, proporcional a las mismas magnitudes: dilatación y esbeltez.

Salv.-Lo variable será solamente la razón de deflexión a estas magnitudes, que no es sino la que resulte de la fracción del vuelo de deflexión a la semiluz.

Aunque, como podréis fácilmente entender por los razonamientos anteriores, no suele ser la dilatación media la que importa, ya que ésta puede variar mucho, sino que, como intentamos aprovechar al máximo la fortaleza de las piezas y ello lo hacemos de acuerdo a la flexión máxima, será la dilatación máxima el valor que más caracterice y más constante sea, por lo que podemos concluir que:

\section{COROLARIO I}

En todos los casos de flexión, la deflexión es proporcional a la dilatación máxima y a la esbeltez, siendo la razón la que resulte de la fracción del vuelo de deflexión a la semiluz, y la de expresar la dilatación media con respecto a la máxima.

Sagr.- ¿Y cómo sabremos si nuestro razonamiento es ahora correcto?, porque antes nos engañamos.

Salv.-No podemos saberlo, simplemente si razonamos con cuidado tendremos menos probabilidades de equivocarnos, aunque si lo aplicamos a un ejemplo resuelto con otro sistema y obtenemos otra solución distinta, sabremos que hemos errado el discurso.

Tomemos pues el caso anteriormente resuelto, el del grabado I.

El alargamiento medio es el máximo, ya que todo él era el mismo. El centro de gravedad es el centro de la semiluz; de donde se deduce que la deflexión es al c'́arto de la luz, como el alargamiento a la mitad del canto. Es decir:

$$
\frac{\delta}{L / 4}=\mathrm{e} \frac{\mathrm{L} / 2}{\mathrm{~h} / 2}
$$

o sea que:

$$
\frac{\delta}{\mathrm{L} / 2}=\frac{\mathrm{e}}{2} \frac{\mathrm{L}}{\mathrm{h}}
$$

que coincide con el resultado que habiamos obtenido ya.

Simp.-_Y si sale igual podemos concluir que no hay equívoco?

Salv.-No, ese es el drama de la Ciencia: sabe cuándo se equivoca pero no sabe cuándo dice la verdad; aunque un razonamiento cuidadoso es más fiable, y cuanto más simple sea lo que se dice, más fácil será no equivocarse en lo que se dice, y en entenderlo.

Pero volvamos a nuestro discurso, y encontremos el valor de la deflexión con un peso en el centro de la luz. Como los esfuerzos de flexión varian gradualmente desde el centro hasta los extremos, asimismo pasa con los alargamientos, por lo que el alargamiento total, que no es sino la cuadratura del triángulo, valdrá tanto como la dilatación máxima por la semiluz partido por dos. Y el centro de gravedad del triángulo está situado al tercio de la base de la media luz, distando del extremo un tercio de la luz, por lo que la deflexión relativa se obtiene de la relación:

$$
\frac{\delta}{L / 3}=\frac{\mathrm{e}_{\max }}{2} \frac{\mathrm{L} / 2}{\mathrm{~h} / 2}
$$

o sea:

$$
\frac{\delta}{L / 2}=\frac{2}{3} \frac{e_{\max }}{2} \frac{L}{h}
$$
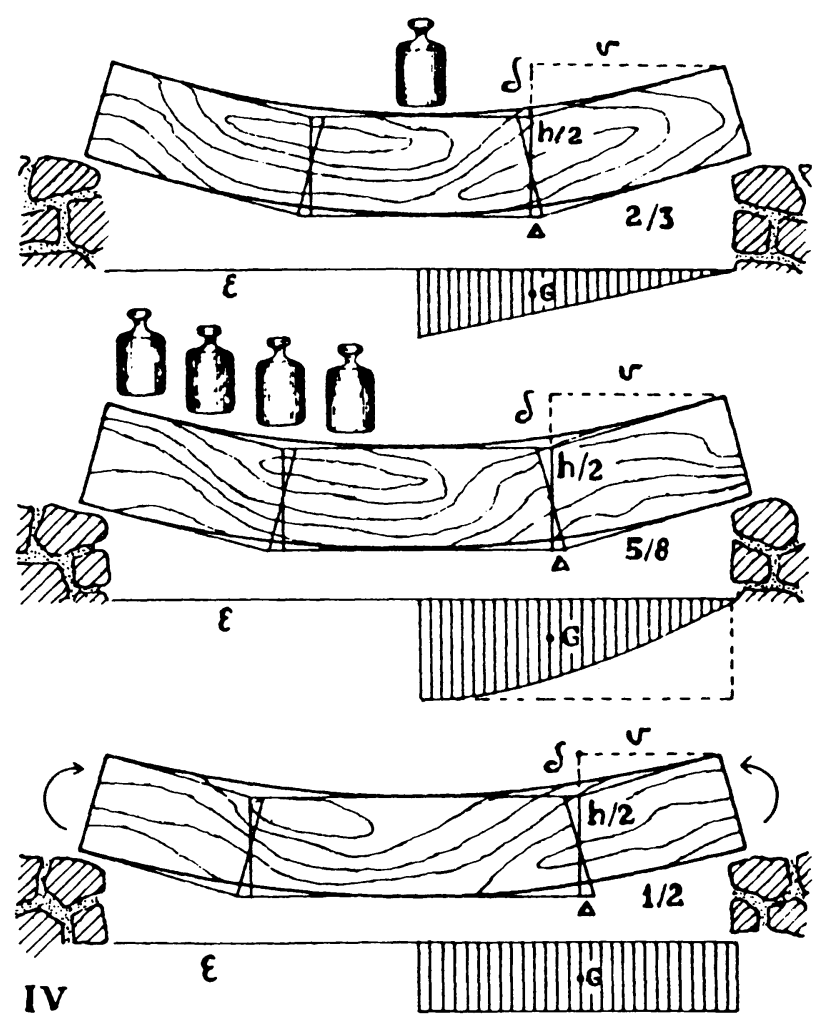

http://informesdelaconstruccion.revistas.csic.es 
Sagr.- ¿Y si el peso estuviese repartido como en el caso de forjados de piso?

Salv.-Entonces, las dilataciones, como los esfuerzos, decrecen desde el centro menos rápidamente que con el peso en el centro, pero al cabo devienen cero en el extremo, y por geometría sabemos que dicha curva es una parábola, y que su área o cuadratura no es sino 2/3 del rectángulo circunscrito y que su centro de gravedad está en los $5 / 8$ de su lado o base, por lo que podremos decir:

$$
\frac{\delta}{\frac{5}{8} \frac{L}{2}}=\frac{2}{3} \mathrm{e}_{\max } \frac{\mathrm{L} / 2}{\mathrm{~h} / 2}
$$

o sea:

$$
\frac{\delta}{L / 2}=\frac{2}{3} \frac{5}{8} e_{\max } \frac{L}{h}
$$

De modo y manera que la deflexión relativa a la semiluz es proporcional a la dilatación máxima y a la esbeltez, y la razón no es otra que la fracción de dilatación media a máxima y la del vuelo de deflexión a la semiluz.

Sagr.-Pero esa conclusión no veo cómo puede sernos últil, ya que no dice cuánto valen dichas fracciones.

Salv.-Mas observad que presenta sin elipsis las magnitudes decisivas: la dilatación máxima, que será la utilizada para elegir la fortaleza de la pieza; y la esbeltez, cuyas características son bien fáciles de apreciar.

Los valores ocultos que decís no son tan importantes, pues ved que, para pesos cualesquiera simétricos respecto a la luz, los esfuerzos están siempre comprendidos entre menguar uniformemente, y no menguar, es decir, podemos asegurar que el primer factor variará sólo entre $1 / 2$ y $2 / 3$, y el segundo entre 1 y $1 / 2$; y que aunque os parezca mucho, fijaros que ambos se mueven conjuntamente; que cuando más la dilatación máxima se parezca a la media, tanto más se parecerá el vuelo de deflexión a la mitad de la semiluz; y que, en resultado, la razón oculta sólo puede variar entre $1 / 2$ y $1 / 3$, que es una mucho menor variación que la de los otros factores. Para tanteos podéis utilizar 0,4 sin temor a equivocaros mucho, al menos para concluir si una determinada pieza, cuya esbeltez es aparente, podrá o no satisfaceros.

Es más, fijaros que ese factor no es oculto, en tanto que es dato del problema propuesto, ya que depende de la situación de las cargas, que son conocidas o forman parte de la propuesta que se nos hace, mientras que las otras - dilatación máxima y esbeltez-son las que debemos aportar con nuestra solución al problema.
Sagr.-Ahora entiendo por qué descomponiais de la manera que parecía caprichosa; que ahora el que tenga que discernir la pieza flectada deberá adoptar valores de las dos segundas para que, con las dos primeras dadas, no supongan deflexión intolerable.

Salv.-En efecto, ese es el propósito de toda expresión: urdir cómo aparezcan en su sitio cada una de las magnitudes que intervienen y que se vea cuál es su papel, y cómo influyen unas sobre otras.

Sagr.-Pero para una misma deflexión, el que sea un sólo peso en el centro, produce más giro en el apoyo, lo que puede perjudicar a las piezas o al muro, y es el vuelo de deflexión el que permite obtenerlo.

Salv.-No sólo eso, sino que entiendo que el valor de la deflexión relativa, ya que el vuelo de deflexión varia entre muy estrechos límites, no es sino una medida aproximada del giro. Que lo que afecta a los tabiques, revestidos y carpinterías no es el descenso, ni siquiera el descenso relativo, sino el ángulo que se ve obligado a seguir, $y$ en ese forzamiento la medida puede ser la deflexión total o el ángulo local, pero como ambas son éstrechamente proporcionales -ya que el vuelo de deflexión que los relaciona no varía sino entre $L / 2$ y $L / 3$, y aún diría que como los pesos existen siempre y es una parte importante de la carga, poco se diferencia de 4 décimas - hablamos del uno por el otro, y a ambos cubre el limitar las deflexiones al 400'avo de la luz.

Pasemos pues a mejorar nuestro propósito, tratando de modo más general alguna de las consideraciones de la Proposición I, y ésta será la de sección simétrica.

Cuando la sección no es simétrica, el mismo esfuerzo no permite que las fibras extremas alcancen, al mismo tiempo, la misma tensión, ya que no se encuentran igualmente separadas del centro de gravedad, de forma que cuando una llega a la resistencia, la otra está en situación mucho más desahogada, y, si ambas resistencias a tensión y compresión son iguales, una de las dos quedará desaprovechada; pero ese no es el objeto de nuestro discurso, que es el de ver cómo se alterarían las conclusiones anteriores por sección no simétrica.

Tomemos pues una sección con cabeza superior más ancha que la inferior, de forma que el centro de gravedad esté, no al centro, sino más arriba. En el grabado siguiente podréis ver que no otra cosa se altera sino que el triángulo semejante al de deflexión y semiluz es el de la dilatación y parte del canto tensionado, que no coincide con medio canto, pero que en los ejemplos anteriores podíamos también denominarlo como la parte en tensión. 

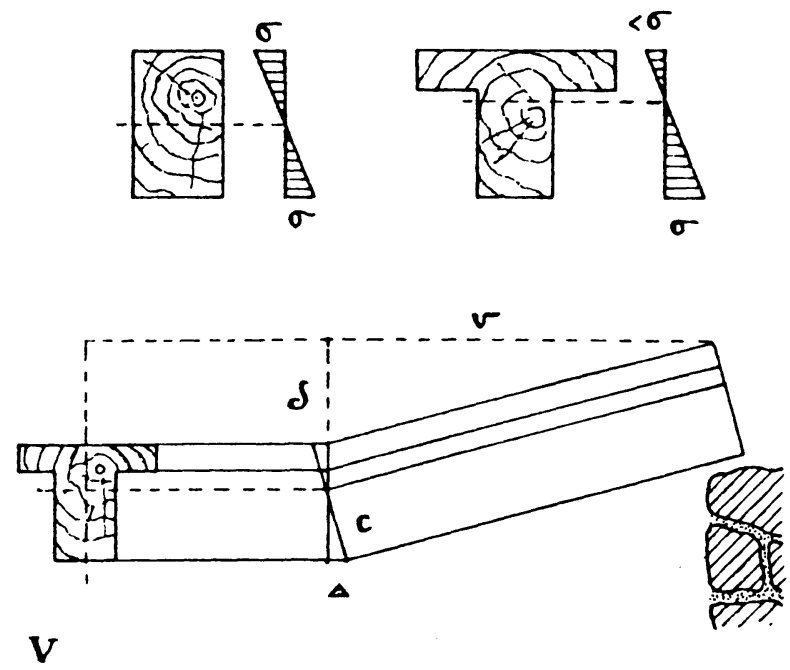

Todas las conclusiones pues se mantendrán, y mientras la sección sea constante, lo será dicho canto tensionado, pudiendo volver a considerar todo el alargamiento en su c.d.g.

Por lo tanto, podemos concluir que:

\section{COROLARIO II}

En toda pieza simplemente presentada, de sección constante y material elástico, la deflexión relativa a la semiluz es directamente proporcional a la dilatación/acortamiento máximo y a la esbeltez, y la razón es la de la dilatación/acortamiento máximo a medio; la de vuelo de deflexión a semiluz; y la de canto en ten. sión/compresión a medio canto.

Sagr.-Me temo que me vais a decir que el nuevo valor no puede variar demasiado.

Salv.-Asi es, porque no es posible que sea inferior a medio canto, y aún en las piezas más desproporcionadas en forma de T con cabeza exagerada, la parte traccionada no puede ser superior al canto total; lo razonable es pues, en una estimación previa, suponer como entre 70 y 80 centésimos del canto. Pero no hay que olvidar que debe tomarse un valor del que estemos seguros que no se supera, porque cuanto mayor sea la parte tensionada menor será la deflexión, y toda estimación segura debe tirar por lo bajo; de lo que se deduce que el valor mitad del canto tiende siempre a asegurar los resultados.

Sagr.-A lo que se ve, la regla de la deflexión siempre es la misma. No podemos hacer casi nada para alterarla.

Salv.-Siempre que adoptemos expresiones atinadas lo será, que ese es el objetivo de la Ciencia.
Y pasemos ahora a mejorar nuestros planteamientos, cuestionando otra magnitud.

Sagr.- ¿Podremos tomar el cambio de material, pasando a uno no elástico o al hormigón?

Salv.-Nos faltaría antes una cuestión previa. Hasta ahora hemos tomado sección constante, y no hemos dudado en adoptar que las dilataciones eran proporcionales a los esfuerzos; antes de adentrarnos en el hormigón será bueno detenernos en esta cuestión: en las piezas de canto constante en las que las dilataciones no sean proporcionales a los esfuerzos.

Simp.-No se me alcanza a ver cómo pueda ser.

Sagr.-No puede ser otra cosa sino el ancho.

Salv. - El ancho es; que no es manera habitual de proceder en madera, pero sí en hierro u hormigón, de forma que se puede proceder con piezas de sección con fortaleza ajustada en cada punto. Así, si el esfuerzo desciende, se disminuye el ancho para encontrar el mínimo material que satisface la resistencia.

Sagr.-¿Y ello, en qué alterará nuestras conclusiones?

Salv.-En nada realmente, que el corolario anterior es en este caso igualmente válido, sólo que antes, para la relación de alargamiento medio y máximo y para el centro de gravedad de los alargamientos, podríamos tomar la curva de esfuerzos, mientras que lo riguroso seria tomar la de alargamientos. Mientras la sección sea constante las dos son proporcionales, y sus cualidades coincidirán en valor; pero si la sección varía, habrá que obtenerla. Aunque a decir verdad, si vamos a proceder ajustando la fortaleza, tendremos que tenerlo hecho, y así para la deflexión no será menester cálculos nuevos.

Simp.-Pero las curvas de alargamientos pueden ser más variopintas que las de esfuerzos, y no podemos conocer de antemano cuál es la variación de la razón antes aludida.

Sagr-Casi estaría tentado de deciros lo contrario, porque si no he entendido mal, si se intenta el ajuste de fortaleza de modo que aún variando los esfuerzos mucho, siempre que se tenga una sección estricta, las tensiones y los alargamientos estarán circundando siempre un valor, por lo que serán menos variopintas.

Salv.-El señor Sagredo está en lo cierto. En el caso de un trabajo cuidadoso, sea cual sea la curva de alargamientos y de esfuerzos (ved grabado siguiente), si se ajusta la fortaleza, el alargamiento será único, por lo que retrocederíamos al primero de los casos que estudiábamos, no como única flexión, sino como único 


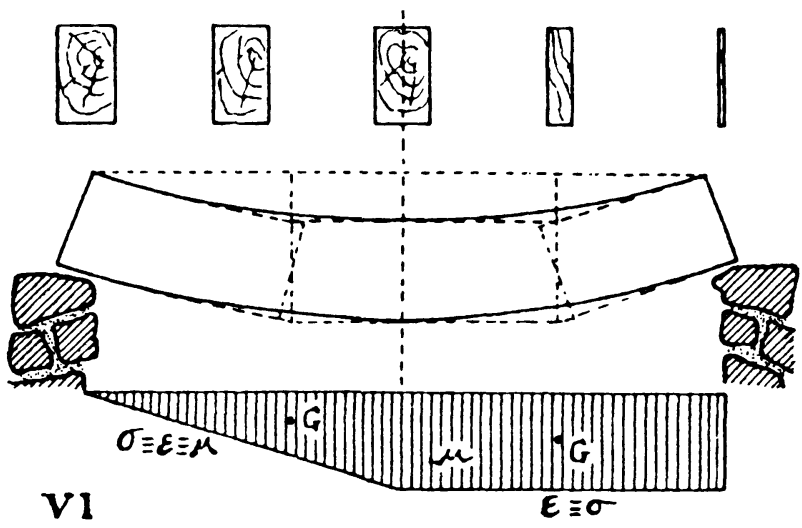

alargamiento, y la deflexión tendrá una razón más cerca de $1 / 2$ que de $1 / 3$, y quedándonos seguros diría que el valor $1 / 2$ es el más razonable.

Así puede establecerse el siguiente

\section{COROLARIO III}

En toda pieza simplemente presentada, de canto y centro de gravedad constante, la deflexión relativa a la semiluz es directamente proporcional a la dilatación/contracción máxima a media, y a la esbeltez, y la razón es la de vuelo de deflexión a semiluz, y la de canto en tensión/compresión a canto total.

Así que ajustar la sección al esfuerzo es algo encomiable, puesto que el conocimiento está, además de goce para el espíritu, para disponer en las cuestiones económicas ni menos de lo necesario ni más de lo suficiente, pero sin desatender otras cuestiones, que si por ajustar la resistencia acentuamos la deflexión, podemos ir en contra de nuestras obligaciones.

Fijémonos que una pieza de peso distribuido, poseía una deflexión de valor:

$$
\frac{\delta}{\mathrm{L} / 2}=\frac{\mathrm{e}_{\max }}{2,4} \frac{\mathrm{L}}{\mathrm{h}}
$$

si se disponía sección constante, y una expresión

$$
\frac{\delta}{\mathrm{L} / 2}=\frac{\mathrm{e}}{2} \frac{\mathrm{L}}{\mathrm{h}}
$$

si se dispone sección ajustada, lo que significa un cuarto más de deflexión.

Por lo que si con una sección normal, una esbeltez 12 es satisfactoria, el intento de ajuste al esfuerzo nos obligaría a tomar la pieza con una esbeltez 9 , que en muchas ocasiones no puede significar una ventaja sino desventaja.

\section{Sagr.-Podremos pasar entonces al hormigon.}

(c) Consejo Superior de Investigaciones Científicas

Licencia Creative Commons 3.0 España (by-nc)
Salv.-Intentémoslo. Varias son las cuestiones que debemos tener in mente. Primera, que no contando con la resistencia del hormigón a tracción, se dispone -en la parte inferior de los ejemplos que nos traemos - barras de acero, que pueden ajustarse mucho al esfuerzo, aunque esa cuestión ya la hemos visto. Segunda, que ese ajuste -aún el simple cambio de esfuerzo- puede significar en la sección un cambio de la posición del centro de gravedad, y aún siendo una pieza de canto constante no cumpliría la condición antes dicha...

Sagr.-Pero esa cuestión es poco importante, que ya voy comprendiendo vuestra astuta manera de acercaros a la cuestión.

Salv. - En efecto, es muy poco importante, ya que el canto tensionado no puede ser inferior a medio canto, $y$ sólo en rarísimos diseños podemos elevarlo a ochenta centésimos del canto, con lo que el promedio que tendríamos que introducir en la razón aludida no puede ser muy diferente de 60 ó 70 centésimos. Y ello si no disponemos de antemano de una sección con cabeza exagerada, que entonces la situación no variará más que entre 80 y 85 centésimos.

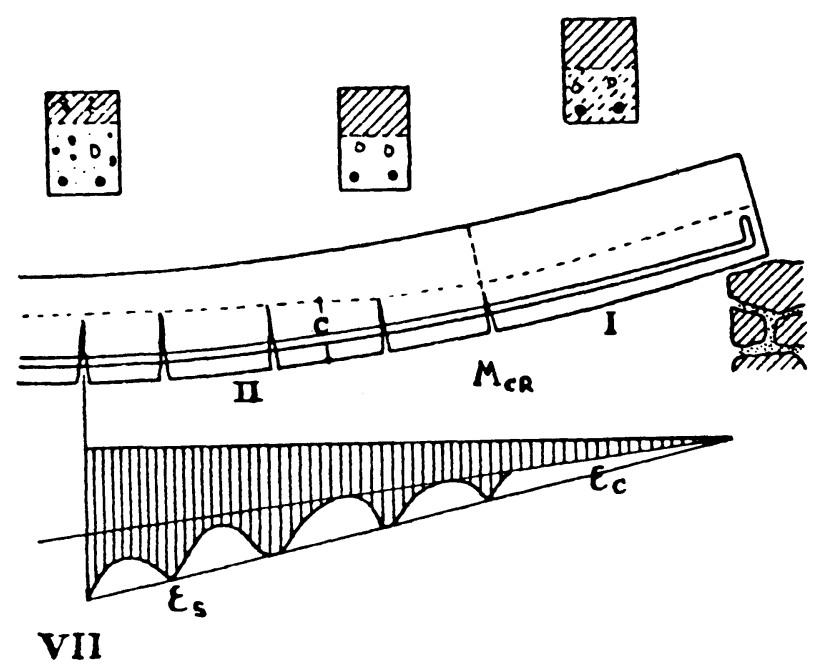

La tercera cuestión, según el grabado adjunto, es que habiendo en las piezas zonas -en los casos en que manejábamos, las cercanas al extremo- con muy poco esfuerzo, casi no importará que haya o no armaduras; que la deformación corresponderá como si de una de hormigón sólo se tratase, y en el resto no quedará más remedio que medirla como si fuera de hormigón armado, de modo y manera que los alargamientos en la fibra inferior no sólo provendrán de considerar una variación del centro de gravedad -ahora impropiamente denominado así, que sería mejor decir el centro de tensiones-, sino que la pieza deberá descomponerse en dos zonas, I y II, cada una de ellas con 
distinta rigidez para, de acuerdo con cada una, obtener los alargamientos.

Y la cuarta cuestión es la peor. Porque aún en los casos en que las zonas en que la fracturación nos obliga a pensar en hormigón y armaduras, entre fractura y fractura, el hormigón asciende sus tensiones, de modo que se asemejan parcialmente a las secciones de la zona I en su rigidez.

Y como quiera que la armadura, por razón de su resistencia, sólo la calculamos en las partes fracturadas, no podemos contar con los cálculos hechos, sino que es preciso abordar cálculos nuevos para ello. Pero eso no alterará lo general de lo ya concluido, por lo que podremos afirmar:

\section{COROLARIO IV}

En toda pieza simplemente presentada, aún de hormigón armado, la deflexión relativa a la semiluz es directamente proporcional a la dilatación máxima, y a la ebeltez, estando afectada la razón con el cociente de vuelo de deflexión a semiluz, dilatación máxima a media, y canto promedio en tracción a canto total.

Sagr-- ¿Y cómo obtendremos esa razón?

Salv.-Ese es el problema, porque ahora su variación es grande, ni siquiera podremos actuar con la curva de dilataciones, sino de cocientes de dilatación a canto tensionado, que es ahora lo que varía.

Y permitidme la licencia - de la que no haré más usode no poder demostraros lo que digo, sino más bien de que os fiéis en argumentos de autoridad, que las investigaciones de Micer Branson y el ajuste a los casos estudiados, que han sido muchos, le han hecho establecer la siguiente conjetura:

\section{CONJETURA DE BRANSON:}

Que la robustez o rigidez de una pieza de hormigón armado, aun variando de sección a sección, puede tomar. se como un promedio, constante e igual a la que tendría como hormigón armado, más una fracción de 10 que resta hasta la correspondiente a sólo hormigón, fracción que se estima igual al cubo del cociente entre el esfuerzo que fractura el hormigón y el máximo esfuerzo, medido todo en el centro de la pieza.
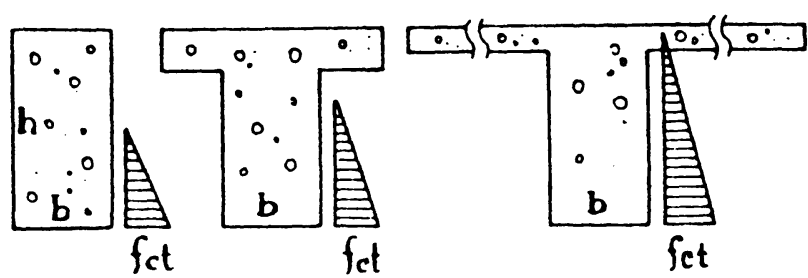

\section{VIII}

(c) Consejo Superior de Investigaciones Científicas

Licencia Creative Commons 3.0 España (by-nc)
Y lo primero que haremos será obtener qué quiere decir, que no es exactamente lo que dice, que es claro y aun tedioso de aplicar, sino cómo podemos hacer transparentes sus repercusiones.

En una sección rectangular, el esfuerzo de fractura es el que nos da la sección, con una tensión inferior de valor $\mathrm{f}_{\mathrm{ct}}$; cómo, según el grabado VIII, la cantidad total de tensión es un área, mitad de la sección total, con una tensión mitad de la máxima, es decir, $\mathrm{f}_{\mathrm{ct}} \mathrm{bh} / 4$; y como la distancia entre la resultante de la parte tensionada y la comprimida es de dos tercios del canto, el esfuerzo de flexión buscado es:

$$
M_{c r}=f_{c t} b h^{2} / 6
$$

Si imaginamos por un instante que estudiamos el caso de una pieza simplemente presentada en los extremos, con peso distribuido, el máximo esfuerzo de flexión es $M=q^{2} / 8$, por lo que el cociente de Branson es:

$a=\left(\left.\frac{M_{c r}}{M}\right|^{3}=\left(\left.\frac{f_{c t} b h^{2} / 6}{q L^{2} / 8}\right|^{3}=\left(\left.\frac{f_{c t} b}{6 q / 8}\right|^{3},\left(\frac{L}{h}\right)^{6}\right.\right.\right.$

Si realizamos las mismas operaciones con una sección en $T$, la cantidad total de tensión es la del total casi del nervio, con una tensión media mitad de $f_{c t}$, es decir, $f_{\mathrm{ct}}$ bh/2; aunque incluso con cabezas muy exageradas no se puede llegar a 80 centésimos de esa cantidad.

La distancia entre el centro de tensiones y compresiones no puede en ningún caso exceder de $2 / 3$ del canto total, por lo que el esfuerzo de flexión buscado no puede exceder de:

$$
M_{c r}<f_{c t} b h^{2} / 3,3
$$

y yo diría que un límite práctico es:

$$
M_{c r}=f_{c t} b h^{2} / 4
$$

Considerando el caso anterior de pieza presentada con peso distribuido quedaría:

$a=\left(\frac{M_{c r}}{M}\right)^{3}=\left(\frac{f_{c t} b h^{2} / 4}{q L^{2} / 8}\right)^{3}=\left(\frac{f_{c t} b}{4 q / 8}\right)^{3},\left(\frac{L}{h}\right)^{6}$

Admitiréis conmigo que en los casos de piezas de forjar pisos, en las que la cabeza, aun siendo importante, no lo es tanto, se encontrará entre ambos casos [1] y [2], y en primera aproximación podemos tomar:

$$
a=\left(\frac{f_{c t} b}{5 q / 8}\right),\left(\frac{L}{h}\right)^{6}
$$

Simp.-No veo para qué necesitamos todo ello y la generalidad de las conclusiones que podamos obtener.

Salv.-Por lo que respecta a la variable cantidad "a" es general, aunque entendamos el valor 5 variable, desde 
6 para el caso de sección rectangular; hasta 4 para el de sección en $\mathrm{T}$ con cabeza muy exagerada; y todos los casos posibles del grabado final son intermedios entre ambos.

El coeficiente q/8 permite considerar sólo el caso de carga repartida; pero cualquier otro caso de carga - simétrica con la luz - bastaría tomar para q/8 el valor $M_{\text {max }} / L^{2}$; pero como los pisos tienen una fracción del peso -el propio - en forma distribuida y aún en muchos códigos se indica que debe considerarse toda, no será un desatino mantenerlo.

El coeficiente $f_{c t} b$ es como la resistencia de toda la fibra inferior, con lo que entenderemos mejor muchas otras situaciones de las previstas.

Mientras ese esfuerzo $\mathrm{M}$ sea menor del que fractura la sección (de $\mathrm{M}_{\mathrm{cr}}$ ), según el grabado que os muestro, todo el hormigón está sin fracturar, y su rigidez se mide sin armadura, de acuerdo con el Corolario I; si M sobrepasa el valor $M_{c r}$, la rigidez disminuye hacia el valor del hormigón fracturado, pero tan rápidamente que cuando $M$ es el doble de $M_{c r}$, ya ha salvado más del 85 centésimas de dicho intervalo; y si $M=3 M_{c r}$, más del 95 centésimas.

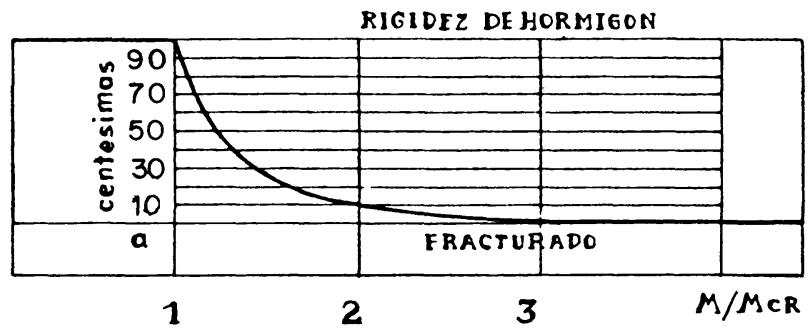

IX

Importará pues saber si $M$ es o no mayor que $M_{c r} y$ cuánto más, en los casos que estudiemos, para saber de antemano la complejidad de los cálculos; y la forma será estimar el valor de "a", que no es sino las centésimas que nos quedan para llegar a la rigidez como si de hormigón armado fuera toda la pieza.

Sagr.-Pero dependiendo de la sexta potencia de la esbeltez, variará muy rápidamente.

Salv.-Bien decís, porque por muchos valores que puedan adoptar $f_{c t}$, b y q, para cada combinación de ellos, con mover ligeramente la esbeltez, su influencia quedará prontamente amortiguada, de manera que sólo en una gama estrechamente acotada, la conjetura de Branson hace retocar los cálculos simples.
Salv.-Para ello he confeccionado el cuadro que os muestro.

CUADRO I

\begin{tabular}{|c|c|c|c|}
\hline \multirow[b]{2}{*}{$\begin{array}{l}\text { TIPO } \\
\text { SECCION } \\
b=10 \mathrm{~cm}\end{array}$} & \multirow[b]{2}{*}{$\square_{b}^{b}$} & \multirow[b]{2}{*}{$\coprod_{b}^{7 b}$} & \multirow[t]{2}{*}{$30 \mathbf{b}$} \\
\hline & & & \\
\hline$q=200$ & $20-25$ & $22-27$ & $25-34$ \\
\hline$q=900$ & $14-18$ & $16-21$ & $18-29$ \\
\hline$q=600$ & $11 \cdot 15$ & $13-17^{*}$ & $15-20$ \\
\hline \multicolumn{4}{|l|}{$b=20 \mathrm{~cm}}$. \\
\hline$q=200$ & $28-$ & $31-$ & $35-$ \\
\hline$q=400$ & $20-25$ & $22-29$ & $25-36$ \\
\hline $9=600$ & $15-21$ & $18-24$ & $23-28$ \\
\hline \multicolumn{4}{|c|}{ ESBELTEZ PARA $a=0.05-a=0.01$} \\
\hline
\end{tabular}

En primer lugar, he tomado un ancho $10 \mathrm{~cm}$, que es el usual en piezas para forjar pisos, y ya hemos dicho que una variación de 8 a $12 \mathrm{~cm}$ no importará demasiado. En segundo lugar, hemos reflejado el ancho $20 \mathrm{~cm}$, para tener las piezas dobladas y aún las piezas de jácena normal.

De cada uno presento tres tipos de sección, que no son sino los anteriormente estudiados: el de sección rectangular; el de sección en T con cabeza muy exagerada -que los presento como casos límites-; siendo los reales, intermedios, que los represento como un caso, pero entended bien que todos ellos pueden estar comprendidos entre los otros dos.

En tercer lugar, tomo hasta tres cargas diferentes: de $200 ; 400$ y $600 \mathrm{~kg}$ por metro de pieza, lo que viene a ser como el peso propio, el intermedio cuando se trata de construcción, y el total para el que se destinan los pisos de casas privadas, que en los edificios públicos aún sería más.

El valor que represento en el cuadro no es más que el de la esbeltez, para el que ya sólo quedan 5 centésimas de la rigidez del intervalo entre ambos valores citados, y el de 1 centésima.

Y para entenderlo aún más, deduciremos en detalle uno de los valores.

Sea pues una pieza, ni rectangular, ni de T exagerada, como la del grabado $X$. Su centro, según se puede deducir, se encuentra a 70 centésimas del canto, desde su parte inferior, por lo que la tensión total, cuando llegue a fracturar, será $0,7 \mathrm{hbf}_{\mathrm{ct}} / 2$, y la distancia entre ambas http://informesdelaconstruccion.revistas.csic.es 
$7 \mathrm{~b}$

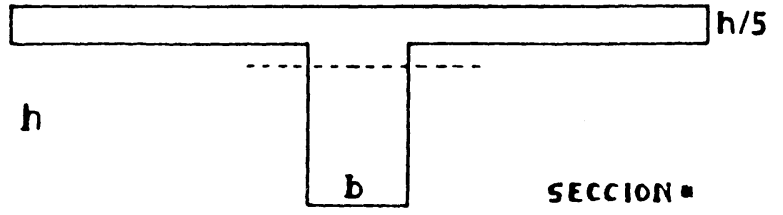

RI6IDE2 DE HORMIOON

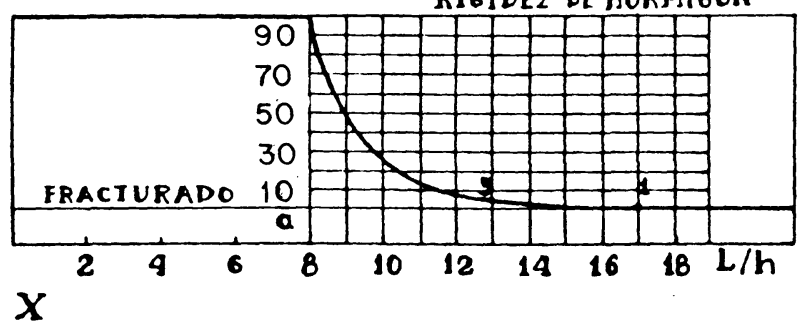

partes no puede llegar a $2 / 3$ de 0,7 h más $0,2 \mathrm{~h}$, es decir, de 2/3 del total, por lo que:

$$
M_{c r}=0,23 b h^{2} f_{c t}
$$

Si tomamos una carga de $600 \mathrm{~kg} / \mathrm{m}$, o bien de $6 \mathrm{~kg} / \mathrm{cm}$, el esfuerzo máximo será $M=6 L^{2} / 8$, de donde el valor $M_{c r} / M$ es $6 L^{2} / 8$ entre $0,23 b h^{2} f_{c t}$, es decir:

$$
\frac{M_{c r}}{M}=\frac{0,31 f_{c t} b}{\left(L^{2} / h^{2}\right)}
$$

Para los hormigones ordinarios, la resistencia a tensión, aún la media, está en proporción sexquiáltera con la de compresión, y para $\mathrm{H}-175$ es $\mathrm{f}_{\mathrm{ct}}=21 \mathrm{kp} / \mathrm{cm}^{2}$. Tomando el ancho de $b=10 \mathrm{~cm}$, quedaría $M_{c r} / M=64,4 /$ $/\left(L^{2} / h^{2}\right)$, de lo que se deduce que para una esbeltez raiz cuadrada de 64,4 , o sea 8 , la pieza presenta fractura, pero para esbeltez 11, el momento ha doblado ya al de fisuración, y para 14, lo ha triplicado, de forma que el valor "a" ya es menor de 5 centésimas, y para esbeltez 18 , el momento ha quintuplicado el de fracturación, de forma que el valor de "a" es apenas una milésima, pudiendo ser despreciado tal factor.

Así se han confeccionado todos los valores del Cuadro I.

Sagr.-Pero dichos valores son casi todos muy pequeños.

Salv.-En efecto, en piezas simplemente presentadas en los extremos, lo habitual -y la buena práctica lo sanciona- es disponer cantos del orden del veinteavo de la luz, al menos cuando las tabiquerias se reciben con yeso, que cuando se calculan con esmero las deflexiones aún está autorizado el tomarlos inferiores.
Sagr.-Así, no es preciso considerar más que la rigidez de las piezas como si estuvieran fracturadas.

Salv.-No exactamente. Con más rigor tendremos que decir que, cuando el ancho es pequeño, el hormigón normal, y estemos considerando el peso total, no será imprescindible complicar los cálculos, debiendo tomar directamente la rigidez de la pieza fisurada; pero observad bien que no digo los cálculos de cualesquiera deformaciones, sino sólo el de comprobar que son aceptables, que adoptar la rigidez fracturada sólo ajusta cuando los cálculos se realizan en piezas de esbeltez que raya en lo intolerable, que sólo en esos casos puede hacerse tal simplificación.

Y notad dos cosas más; la primera, que cuando se tomen piezas dobladas o de $20 \mathrm{~cm}$ y sean en T muy exagerada, y la pieza deba soportar muy poco peso -como es el caso de azoteas y terrazas en zonas cálidas en las que no es de temer la nieve-, su rigidez es mayor, aunque se pueden seguir haciendo cálculos seguros, adoptando la de hormigón fracturado.

Sagr.-Pero, siendo los cálculos seguros, conducirán a tomar un canto mayor del necesario y ello, como en todo, no realizará -el entendido - un buen cumplimiento de sus obligaciones, que son defender un diseño seguro pero sin excederse un ápice.

Salv.-Notad empero que al tomar un canto mayor podrá elegirse el acero adecuado al esfuerzo, resultando una pieza siempre igual de segura, salvo que no posee deflexiones importantes, y ello no se ha conseguido con más material del imprescindible, sino en disponerlo de manera más ventajosa, y que dicha forma no es apenas más costosa, y así cumplirá bien con su cometido; que si intenta proceder con más finura que lo cumplidas que son las hipótesis, podrá, sin saberlo, pasar a situaciones no deseadas, y por ello la prudencia impele a adoptar en estas procelosas cuestiones con algo más de mesura.

Item más, si las deflexiones son importantes en hormigón, lo son fundamentalmente por el transcurso del tiempo, lo que nos obligará a encontrar valores para cada fase de la construcción, incluyendo pesos tan pequeños como el propio, con los que ya no es tan riguroso tomar como rigidez la del hormigón fracturado, y si se adopta así quedan los cálculos muy inflados, previendo deflexiones más grandes que las reales y conduciendo a piezas mucho más holgadas de lo necesario.

http://informesdelaconstruccion.revistas.csic.es 
Así puede pues decirse:

\section{COROLARIO V}

En piezas de forjar pisos, de hormigón armado, de sección en $T$ aún no muy acusada, simplemente presentadas en los extremos, sometidas a peso distribuido cuando las deflexiones rondan los valores inaceptables, éstas son proporcionales a la dilatación máxima y a la esbeltez; y de ser cierta la conjetura de Branson, debe adoptarse para su cálculo el hormigón como fracturado en toda su luz.

Sagr.-Pero aún cuando la rigidez sea parecida a la del hormigón fracturado, no hemos considerado si el margen hasta la del hormigón es tan grande que cualquier pequeño aumento sería muy beneficioso.

Salv.-En efecto, y muy atinada es la observación, y aunque sea sólo por satisfaceros la consideraremos.

Pero ello nos obliga a dar antes un rodeo, porque hasta ahora habiamos tomado la curvatura de la sección como el cociente de la dilatación al canto tensionado, y ello, como habíamos visto en otra jornada anterior (2) no es sino el cociente entre el esfuerzo y la robustez, y ésta no es sino el producto del módulo de deformabilidad por el de momento de segundo orden de la sección, y deberemos detenernos en ésta para resolver vuestra pregunta.

Comenzaremos por una sección rectangular. El momento de segundo orden del hormigón es, como veíamos, bh $h^{3 / 12}$, mientras que una sección en $T$ tan acusada que el centro de gravedad estuviera en su parte superior, daría un momento de segundo orden de $\mathrm{bh}^{3} / 3$, aunque afirmo que en lo real será poco menos que imposible llegar tan siquiera a bh $3 / 4$. De ello, podemos deducir que en las secciones de forjar pisos, el momento de segundo orden estará comprendido entre ambos y, para facilitar los cálculos, si ellos son $0,08{b h^{3}}^{3}$ $0,25 \mathrm{bh}^{3}$, podemos decir que están comprendidos entre $0,10 \mathrm{bh}^{3}$ y $0,20 \mathrm{bh}^{3}$.

Para el momento de segundo orden de la sección fracturada debemos comenzar asimismo por la rectangular; el momento será: $I=b x^{2} / 2 \cdot 2 x / 3+n A(d-x)^{2}$, siendo $x$ la parte comprimida; $A$ el área del acero, $d$ el canto al acero, y $n$ el cociente de módulos de deformabilidad de acero a hormigón. Y como del equilibrio se debe cumplir que la línea ax de la cara superior es el centro, será:

$$
b x \cdot x / 2=n A(d-x)
$$

con lo que el momento de segundo orden es:

$$
I=n A(d-x) \cdot 2 x / 3+n A(d-x)(d-x)
$$

o sea:

$$
I=n A(d-x)(d-x / 3)
$$

como se ve, pues, función sólo del acero, interviniendo el hormigón sólo para dar el valor $\mathrm{x}$.

Pero en variando el valor $x$ con la cantidad o cuantía de acero, y contando con un valor de $n$ en torno a 8 , podemos poner para:

$$
\begin{array}{ll}
A / b h=0,002 & \text { entonces } I=0,64 n A h^{2}=0,01 b h^{3} \\
A / b h=0,004 & \text { entonces } I=0,60 n A h^{2}=0,02 b h^{3} \\
A / b h=0,008 & \text { entonces } I=0,50 n A h^{2}=0,03 \mathrm{bh}^{3}
\end{array}
$$

De modo que los momentos de segundo orden de la sección fracturada pueden hacer bajar la rigidez a valores mitad, cuarta y hasta octava parte; lo que significa la necesidad de establecer valores ajustados a los fenómenos; y la importancia de los trabajos de micer Branson.

Pero si tomamos una sección en T idealmente acusada, el centro estará siempre en la parte superior, y el momento de segundo orden será siempre $I \bumpeq n A d^{2}$, que difícilmente puede llegar a ser en los casos reales tanto, y aunque d sea $0,9 \mathrm{~h}$, un valor probable será:

$$
I=0,7 n A h^{2}
$$

Si confiáis en lo que digo, afirmo que los aceros en este tipo de piezas, y de acuerdo con las esbelteces que pudieran ser sospechosas de deflexiones críticas, están entre $A=0,01$ bh y $A=0,03 \mathrm{bh}$, por lo que los momentos de segundo orden pudieran ser para:

$$
\begin{array}{ll}
\mathrm{A} / \mathrm{bh}=0,01 & \text { entonces } \mathrm{I}=0,70 \mathrm{nA} h^{2}=0,06 \mathrm{bh}^{3} \\
\mathrm{~A} / \mathrm{bh}=0,02 & \text { entonces } I=0,70 n A h^{2}=0,12 \mathrm{bh}^{3} \\
\mathrm{~A} / \mathrm{bh}=0,03 & \text { entonces } \mathrm{I}=0,70 n A h^{2}=0,18 \mathrm{bh}^{3}
\end{array}
$$

De modo que los momentos de segundo orden de la sección fracturada pueden no significar pérdida de rigidez o bajarla a la mitad, y alguna que otra vez a la tercera o cuarta parte, resultando mucho menos importante la determinación en detalle de la rigidez real.

En los casos de secciones de piezas de forjados, intermedias entre ambos anteriores, afirmo que los valores están entre $0,50 n A h^{2}$ y $0,70 n A h^{2}$ necesariamente, quedando: 
Sección fracturada para:

- cargas y cuantias bajas

I de $0,04 \mathrm{bh}^{3}$ a $0,05 \mathrm{bh}^{3}$

- cargas de habitaciones

I de $0,09 \mathrm{bh}^{3}$ a $0,11 \mathrm{bh} \mathrm{h}^{3}$

- cargas y cuantías elevadas I de $0,15 b h^{3}$ a $0,18 b h^{3}$

De modo que cuanto mayor es la carga, menos importancia tiene tomar la rigidez del hormigón fracturado, y sólo en los casos de techos sin peso pudiera ser interesante adoptar cálculos más minuciosos. Que en los casos de pisos de habitaciones, no tomar 5 centésimas de la diferencia entre una y otra no llega a significar ni esas 5 centésimas en los cálculos, y que en las demás cuestiones, como módulo de deformabilidad 0 coeficientes de deflexiones a largo tiempo, tienen estimaciones mucho más groseras, y que la precisión de los cálculos no debe ser superior al del menos fielmente establecido de los pasos y valores intermedios.

Sagr.-Admitiendo pues lo que nos habéis dicho, aunque si he de ser sincero más me he fiado de vos que seguido a pies juntillas los razonamientos, debemos pues, en hormigón para las piezas de forjados, tomar como fracturado al hormigón; pero aun en ese caso, no caigo cómo considerar el cálculo de la deflexión.

Salv.-Es cierto que en los casos de sección rectangular no sólo es imprescindible tomar una situación mixta, sino que además la determinación de la sección fracturada no ha sido necesariamente vista en el cálculo de armaduras, y ello exige nuevos cálculos para deflexiones.

Pero para piezas en T, como las de los forjados, todo es más simple.

Si convenis conmigo que lo que intentamos saber es si será posible disponer cantos inferiores a pongamos el veinteavo de la luz, sin que existan deflexiones inaceptables, supongamos que lo disponemos así. Para peso distribuido el máximo momento es $M=q L^{2} / 8, y$ en una estimación previa la armadura será la de suministrar una fuerza $U=M / 0,9 \mathrm{~h}$, de lo que resulta $\mathrm{U}=2,7 \mathrm{qL}$.

Pero si en la cara superior del forjado existe no menos de 3 ó $4 \mathrm{~cm}$ de hormigón, y las piezas se disponen cada 50 ó $70 \mathrm{~cm}$ al menos, resultará que un hormigón de resistencia $f_{c d}$ puede dar $U_{c}=f_{c d} \cdot 150$ como poco, y $U_{c}=f_{c d} \cdot 280$ como mucho; bastando con un hormigón de los llamados H-175 para cubrir los $10 \mathrm{~m}$ de luz hasta con pesos habituales en edificios públicos.

Admitiréis pues que tomar como línea de separación entre parte fracturada la que limita el nervio con el ala de la T, ofrece en este caso una exactitud suficiente, y por tanto no tendremos siquiera que hacer cálculos con la sección fracturada, sino tomar directamente la curvatura como la dilatación de acero entre el canto del acero en el nervio.

Simp.-_Pero cómo obtendremos la dilatación del acero?

Sagr.-Me temo que el señor Salviatti nos va a proponer alguna regla asimismo sencilla.

Salv.-Naturalmente, porque sólo si las reglas son sencillas, sencillo es su cumplimiento, que de las cosas que se hacen por primera vez es justo acudir a la teoría, y hacer cálculos minuciosos, que entonces son los cálculos basados en las reglas generales los que nos dan una guía de lo que acontecerá; $y$ aún en ese caso deberemos luego comprobarlo en la realidad, que no podemos fiarnos de nuestros conocimientos y nuestras reglas, que sólo son modelos imperfectos.

Pero de lo que llevamos tiempo haciendo, debemos esforzarnos en poderlo representar de forma sencilla e inteligible, que sólo en ese caso sabremos aplicarla; y que en los casos habituales todo calculista deberá saber lo que sale, y si los cálculos no coinciden con lo que sabia, debe sospechar que son los cálculos equivocados; y que no es prudente dar reglas complicadas para lo que está suficientemente conocido.

Y la regla para obtener la deformación del acero será la de dividir también -en estos casos- el esfuerzo entre la distancia entre el acero y el centro de la parte de la cabeza de hormigón, y con esa fuerza y con el área sacaremos la tensión y aún la dilatación.

Sagr.- ¿Pero esos cálculos no han sido hechos ya para determinar el acero?

Salv.-Os habéis anticipado a mis pensamientos, porque, en efecto, ya han sido hechos, y de adoptar un acero ajustado al esfuerzo, siempre obtendremos el mismo valor de dilatación, y afirmo que con acero A400 no se alejará mucho de una milésima; pero todo exceso, aunque sólo sea para encontrar armaduras de sec-

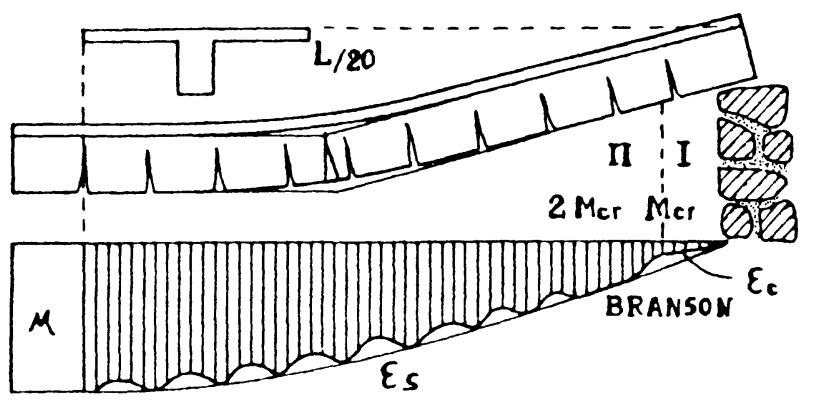

X I

http://informesdelaconstruccion.revistas.csic.es 
ción convenida, redundarán en descender la dilatación y con ello la deflexión. Y que si, según el Cuadro I, la esbeltez que elegimos quintuplica el esfuerzo máximo al que fractura la sección, y los esfuerzos no descienden a cero en el extremo, sino parabólicamente, es a una distancia de un canto del extremo cuando se alcanza la fractura, y sólo un canto es la zona de hormigón, en la que la deformabilidad es como sólo hormigón, y sólo en los dos otros cantos primeros, de los veinte, sería necesario corregir nuestros cálculos con la conjetura de Branson, y que, al ser poca la dilatación y poco el vuelo de deflexión de esta parte, no hay inconveniente en ignorarlo, y ese es el grabado XI.

Sagr.-Pero aún con ello, observo que falta el caso de ser el hormigón precomprimido, y de las piezas no sólo presentadas, sino de las firmemente entregadas.

Salv.-En efecto, pero mirad que las precomprimidas poseen un valor $f_{c t}$ como vez y media el antes considerado, aunque al estar precomprimido contará como $f_{c t}$ su descompresión, lo que eleva el total a ocho o diez veces lo que veníamos considerando, pero exis- tiendo hormigón in situ en ancho inferior, sería éste el que fracture antes, por lo que se compensa todo, resultando un factor $\mathrm{f}_{\mathrm{ct}} \mathrm{b}$ no muy diferente. En cuanto a las continuas, sólo influyen en el coeficiente q/8, que pasará a q/12 ó q/16, que se compensa con la mucha mayor esbeltez que se pretende con esas piezas; por lo que, como la jornada ha sido larga y cansada, dejaremos para mañana comprobar en detalle la siguiente proposición:

\section{PROPOSICION II}

En piezas de forjados de piso, de hormigón armado o precomprimido, de sección en $T$, aún las no demasiado acusadas, simplemente presentadas o entregadas eficazmente en algún extremo, como las continuas, sometidas a peso distribuido, cuando las deflexiones rondan los valores inaceptables, el valor instantáneo de la deflexión - de ser cierta la conjetura de Bransones directamente proporcional a la dilatación máxima del acero, a la distancia del vuelo de deflexión según la curva de dilataciones, a la fracción de canto tensionado respecto a la mitad del canto y a la esbeltez.

\section{publicación del IETcc / CSIC}

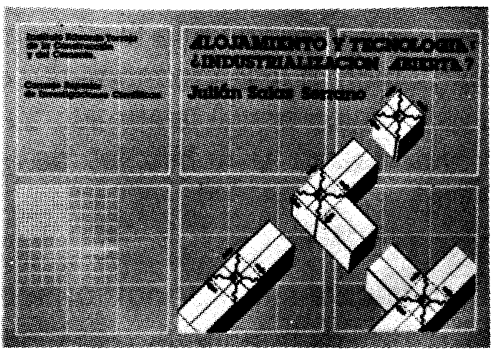

La experiencia nacional en construcción industrializada en la última década, aunque no abundante, puede resultar paralizante. Como reacción, este trabajo trata de elaborar y ordenar la información que, pegado al terreno, se ha acumulado durante los sesenta.

Auscultando tendencias avanzadas en otros paises y apoyándose en nuestra realidad cotidiana, el autor de este trabajo apuesta por la industrialización, presintiendo un futuro con pocos puntos en común con lo que en general, hasta bien reciente, se ha conocido como construcción industrializada.

Se abre el trabajo con un prólogo del Profesor Ciribini en el cual, con rigor conceptual y desde su rica experiencia, analiza algunos de los conceptos más polémicos del fenómeno de industrialización del sector construcción. El autor recopila un conjunto de aportaciones de maestros de la arquitectura al lento proceso de evolución tecnológica y conceptual continuando con un intento de respuesta realista a la interrogante que flota en el sector nacional: ¿réquiem por la industrialización?

Se dedican otros capítulos a revisar lo realizado y a encarar el futuro inmediato: el concepto de componente, su repercusión económica, la dificultad del proyecto a base de productos industriales, la nueva vigencia embrionaria de la construcción por medio de catálogos, los conceptos de flexibilidad e intercambiabilidad desde una óptica práctica... Especial atención dedica este trabajo a la exposición de lo que podíamos llamar nuevos derroteros de la coordinación dimensional, aportando una visión actualizada y pragmática de los enfoques más implantados en Europa.

Las siempre problemáticas interrelaciones entre normativa, calidad e industria se detallan de forma documentada y realista. Finaliza el trabajo con unas reflexiones dirigidas al ámbito empresarial y un esbozo de «reglas de juego" que faciliten al subsector la salida del uimpasse» actual.

Un volumen encuadernado en rústica, de $24 \times 16,5 \mathrm{~cm}$, compuesto de 160 páginas, con 109 figuras, 19 tablas y 86 referencias bibliográficas. Madrid, junio, 1981 . 